Anatomy and Physiology | Editorial

\section{Idiopathic Inflammatory Myopathies (IIMs)}

\section{Adel Ekladious}

\author{
Submitted: 21 February 2021 \\ Approved: 28 February 2021 \\ Published: 28 February 2021
}

Address for correspondence: Adel Ekladious, Associate professor of medicine, Faculty of health and medical sciences, University of Western Australia, G11 M Block UWA health, Campus (QF11) Monash.

E mail ID: adel.ekladious@health.wa.gov.au.

How to cite this article: Ekladious A. Idiopathic Inflammatory Myopathies (IIMs). G Med Sci. 2021; 2(1): 038-041.

https://www.doi.org/10.46766/thegms.anp.21022105

Copyright: (C) 2021 Adel Ekladious. This is an Open Access article distributed under the Creative Commons Attribution License, which permits unrestricted use, distribution, and reproduction in any medium, provided the original work is properly cited.
Diagnosis of inflammatory myopathy are usually based on clinical examination and histopathology of the muscles [1] and muscle biopsy, usually guided by PET-CT [2]. This is not uncommonly presented by extramacular signs like interstitial lung disease, dysphagia, few skin signs, loss of weight, arteritis, cardiomyopathy, Pulmonary hypertension, respiratory failure, and cancer [2].

With improvement of laboratory testing, identification of antibodies titers, has started to play an important role in diagnosis, treatment, monitor progression of the disease, and importantly prognosis, and what is likely that patient will develop cancer and surveillance for cancer [3].

Currently standard practice is to measure serum creatinine kinase and muscle specific and muscle associated auto antibodies [4]. Having said that, using different techniques to detect antibodies is affecting sensitivity and specificity [5]. Immunoprecipitation is considered the most accurate test but not available in most medical centers. Immunofluorescence is available but requires experienced staff and carry a risk of variability in different laboratories [6]. Line blot is widely used recombinant antigens and can test multiple antigens at the same time but less accurate for few antibodies including HMGCR, anti J1 and anti-0, which can be tested separately in addition to myositis line blot [7].

\section{Idiopathic inflammatory Myopathies}

These include five main groups, which encompass, inclusion body myositis, anti-synthetase associated myopathy, Dermatomyositis, overlap syndrome of myositis and immune mediated necrotizing myopathy [8]. Muscle biopsy play a role to exclude metabolic myopathy, congenital muscular dystrophy especially limb girdle muscular dystrophy, toxic myopathy and inflammatory myopathy. Of note, myositis specific antibodies occur only in myositis and myositis subtype. On the contrary, Myositis associate antibodies are not limited to myositis' but also positive in collagen diseases. Interpreting autoantibodies is important for clinical diagnosis and help to avoid invasive testing like muscle biopsy [9].

Diagnosing dermatomyositis does not need muscle biopsy. Antibody testing in dermatomyositis are sensitive and specific not only to diagnose the disease but also estimate the risk of associated malignancy [10], which includes anti-TIF-Y, anti-NXP2, dermatomyositides with other autoantibodies that do not carry a risk of cancer except if the patient is above 50 (age relating cancer).

Cancer of patient with positivity for antisynthetase (anti-Jo1) is ethnic specific. The people from Europe and America do not have a risk of cancer, whereas Asian have a risk of cancer specially if they are seropositive for anti-Jo1, anti-PL-12 or anti-SAE1 in addition to sero negativity [11].

All patients with dermatomyositis and aged 50 will need cancer surveillance, which includes CT chest, abdomen, pelvis, colonoscopy and mammography for females and ultrasound testis for men. There is no specific cancer for dermatomyositis and all paraneoplastic cancer can occur [12]. 


\section{I am going to discuss antibodies for diagnosis of} myopathy-

\section{Myositis specific antibodies}

Anti-TIFI-Y, anti-MDA5, anti-SAE1, anti-NXP-2, anti-MI-2 are associated with dermatomyositides. There is no need to biopsy for confirmation because these antibodies are phenotype specific.

\section{MDA5}

Can be detected in Asymptomatic females, mechanic hands, classic rash of dermatomyositis, gottron papules Poly arthritis and interstitial lung disease [13].

\section{NXP2}

Associated with cancer, profound weakness, and calcinosis.

\section{MI2}

Associated with cancer, profound weakness, and calcinosis.

TIFi-Y

Classic rash of dermatomyositis, cancer, dysphagia, and common in children.

\section{SAE1}

Dysphagia, cancer, rash, and interstitial disease in Asian females.

\section{Jo1}

Interstitial lung disease, signifiable weakness, cancer, arthritis, and rituximab responsive if started early [13].

\section{PL7}

Weakness, cancer, and rituximab responsive if started early.

\section{PL12}

Interstitial lung disease, cancer, and moderate weakness.

\section{RNP}

Mixed connective tissue disease.

\section{KS}

Interstitial lung disease.

EJ Interstitial lung disease, and anti-synthetase syndrome.

PM-Scl

Scleroderma, and SLE.

\section{RNP}

Mixed connective tissue disease.
SSA (Ro52 and Ro60) and SS B(la)

Systemic sclerosis's, Sjogren's syndrome, antisynthetase syndrome, and rheumatoid arthritis.

\section{Immune- mediated necrotizing Myopathy}

Common in females, characterized by gradual onset, axial weakness, dysphagia, profound symmetrical weakness, affecting proximal muscles of arm and leg, muscular wasting and elevated creatinine kinase, could be seronegative, or seropositive for HMGCR, patient are at risk to have cardiomyopathy, cardiomyopathy in immune mediated myopathy signals poor prognosis even after treatment with immunosuppressive medication, patient should be screened for all cardiac risk factor and receive treatment for primary prevention [13], catenin kinase should be checked before and after treatment with statin, especially in patients who are sero positive for HMGCR.

This will differentiate between statin induced myopathy and relapse of immune mediated necrotizing myopathy [15]. Patients should be advised against reintroducing of statin [16].

Dysphagia is common in all types of myopathy, also not uncommon in myopathy due to systemic sclerosis, patients who tested positive for anti SPR, are at high risk to develop dysphagia and aspiration pneumonia.

\section{Inclusion body myopathy}

Characterized by gradual onset of Asymmetrical weakness of deep finger flexors, proximal weakness of legs mainly quadriceps, and fascial muscles and dysphagia seropositivity for $-1 \mathrm{AcN}$, signals high mortality, mild weakness bulbar weakness and mild affection of proximal arm weakness, usually affect males more than females, and increased associated autoimmune diseases, shoulder muscles can be affected, important differential diagnosis to be ruled out is limb girdle muscular dystrophy [14].

Inclusion body myopathy is incurable disease, patient should be counselled once the diagnosis had been confirmed, and advanced care plan should be in place including noninvasive ventilation, and nutrition.

\section{Anti-synthetase syndrome}

Characterized by gradual onset, mechanics hand, arthritis, cardiomyopathy, and Raynaud's phenomenon. Proximal muscles are mainly affected associated with dysphagia, weak extensor neck muscles, weakness usually symmetrical, and a bundle of antibodies can be found in antisynthetase and signal phenotype. For exampleAnti-Jo1 commonly associated with cancer (in Asian population) and aggressive interstitial Lung disease in Caucasian [15], and PL12 interstitial lung disease. 


\section{Treatment}

Steroid is the corner stone although response are not equal in all subtypes, steroid sparing agent should be introduced to avoid long term complication of steroid, patient who test positive for Anti-HMGCR and anti SRP should be treated with Rituximab, patient with dermatomyositis and positive for anti- Jo1 respond will to Rituximab, as mentioned before inclusion body myositis is refractory to any treatment $[16,17]$.

In conclusion, testing for autoantibodies should be a standard of practice in 2021 for managing idiopathic inflammatory myopathies as it can diagnose the phenotype, prognosis, response to treatment, rule out limb girdle myopathy, diagnose phenotypes of anti-synthetase syndrome, myopathy is a disease which should involve, neurologist with interest in myopathy, immunologist for interpretation of antibodies, respiratory physician, cardiologist, rheumatologist and geneticist [19].

\section{References}

1. Dalakas MC, polymyositis, dermatomyositis, and inclusion body myositis $\mathrm{N}$ England J MED (1991)325(21);1487-98, DOI: 10.1056/ NEJM1991112325213252017.

2. Marie I, Hachulla E, Cherin P, Dominique S, Harton -P-Y, Hellot M-F, et al interestial lund disease in polymyositis and dermatomyositis, Artheritis Rheum (2002) 47(6); 614-22 DOI: 0.1002/art.10794,

3. Ichimura $\mathrm{Y}$, Matsushita $\mathrm{T}$, Hamaguchi $\mathrm{Y}$, Kaji $K$, Hasegawa M, Tanino $Y$, et al. Anti NXP2 autoantibodies in adult patients with Idiopathic inflammatory myopathy: possible association with malignancy. Ann Rheum Dis (2012) 71-3; DOI: 10.1136/annheumdis-2011-200697.

4. Black HR, Quallich H, Careleck CB, racial differences in serum creatinine kinase levels. Am J MED 1981. Med 1986: 81:479-487

5. Hengstman GJ, Van Engelen BG, van Venrooij WJ. Myositis specific autoantibodies changing insight in pathophysiology and clinical associations, Curr Opin Eheumatol, 2004; 16; 692-699.

6. N J. Mchugh, SL. Tansley, Autoantibodies in myositis, Nat, Rev, Rheumatol, 14 (2018), PP-3027.
7. Z. Btteridge, N. Mchugh, Myositis- specific autoantibodies an important tool to support diagnosis of Myositis, J intern. Med, 280(2016) PP.8-23.

8. O. Benveniste, W.Stenzel. Y. allenbach. Advances in serological diagnostics of inflammatory myopathy, Curr, Opin. Neurol, 29 (2016), PP. 662673.

9. M. Mahler, F.W. Fller, M J Fritzler Idiopathic Inflammatory Myopathy and the antisynthetase syndrome: A comprehensive review -Autoimmune, Rev, 13(2014), PP.367-371.

10. Y. Allenbach, A.L. Mammen, W. Sties Zanviesenzel, O. Benveniste, Immune mediated Necrotizing Myopathies working group, 224th ENMC, international workshop; Clinical sero- pathological classification of immune mediated necrotizing myopathies Zandvoort, The Netherlands-14-16 october 2016, Neuromuscul, disord, 28(2018), PP 87-99.

11. Y.Allenbach, J.Keraen, A-M Bouvier, Jooste, N. CHamptiaux, B. Hervier, et al. High risk of cancer in autoimmune necrotizing Myopathies: Usefulness of Myosities specific antibody Brain, 139(2016). PP 2131 - 2135.

12. Aussy A, Boyer O, Cordel N. dermatomyositis and immunomodulated necrotizing myopathies; A window on autoimmunity and cancer, Front Immunol, 2017;8.

13. Tinular MJ, Soffietti R, Dalmau J, et al. Screening for tumours in paraneoplastic syndrome. Report of an EFNS task force, EUR J Neurol 2011;18;19e3.

14. Wang H, Liu H, X Wang Y, L YXQ, CHEN XX. CAI Left ventricular Diastolic Dysfunction in patients with dermatomyositis without clinically evident cardiovascular disease. J Rheumatol. 2014:41: 4.

15. Motley SA, Sidari A, Hildebrand B, Ward IM. Cardiac manifestations of idiopathic inflammatory myopathy treated with rituximab: A single-center case series and review of the literature. J Clin Rheumatol. doi:10.1097/ RHU.00000000000011413. 
16. Grunwald, S.A., Popp, O., Haafke, S. et al. Statininduced myopathic changes in primary human muscle cells and reversal by a prostaglandin F2 alpha analogue. Sci Rep 10, 2158 (2020). DOI: 10.1038/s41598-020-58668-2.

17. Mammen AL. Statin-Associated Autoimmune Myopathy. N Engl J Med. 2016 Feb 18;374(7):6649. DOI: 10.1056/NEJMra1515161. PMID: 26886523.

18. Ritu Valiyil, Livia Casciola-Rosen, Grace Hong, Andrew Mammen, Lisa Christopher-Stine. Rituximab therapy for myopathy associated with anti-signal recognition particle antibodies: a case series.. PMCID: PMC3107255. DOI: 10.1002/ acr.20219
19. Rituximab in the management of idiopathic inflammatory myopathies complicated by interstitial lung disease.Peter Riddell, Ann O’Mahony, Grainne Murphy, John Ryan, Sinead Harney, Vincent Tormey, Michael Henry. European Respiratory Journal 2017 50: PA898; DOI: $10.1183 / 1393003$

20. Management of the idiopathic inflammatory myopathy (IIMS), by $\mathrm{T}$ Nadin. https://wchh. onlinelibrary.wiley.com/doi/pdf/10.1002/ psb. 1762 